\title{
EFEITO DAS RESTRIÇÕES ESPACIAIS DE ADJACÊNCIA NO PLANEJAMENTO FLORESTAL OTIMIZADO
}

\author{
Lucas Rezende Gomide ${ }^{1}$, Julio Eduardo Arce ${ }^{2}$, Arinei Lindbeck da Silva ${ }^{3}$ \\ ${ }^{1}$ Eng. Florestal, Dr., Depto. de Ciências Florestais, UFLA, Lavras, MG, Brasil - lrgomide@ hotmail.com \\ ${ }^{2}$ Eng. Florestal, Dr., Depto. de Ciências Florestais, UFPR, Curitiba, PR, Brasil - jarce@ufpr.br \\ ${ }^{3}$ Licenciado em Matemática, Dr., Depto. de Engenharia de Produção, UFPR, Curitiba, PR, Brasil - arineicls@gmail.com
}

Recebido para publicação: 31/08/2009 - Aceito para publicação: 23/11/2009

\begin{abstract}
Resumo
O objetivo do estudo foi aplicar restrições espaciais de adjacência no agendamento da colheita florestal em um modelo tradicional de planejamento florestal. Foi analisado e comparado o impacto no VPL (valor presente líquido) e na produção volumétrica de madeira. A área de estudo foi composta por 52 talhões de eucalipto. Foram simuladas 254 alternativas de manejo, sendo então criados 4 cenários de agendamento da colheita florestal envolvendo o uso da programação linear inteira, seguindo a formulação pelo modelo tipo I com maximização do VPL e um horizonte de planejamento de 7 anos. O cenário 1 não considerou a adjacência, enquanto que os cenários 2 (URM), 3 (ARM50) e 4 (ARM70) continham restrições de adjacência. Os resultados demonstraram que as restrições de adjacência reduzem o VPL em 3,74\%, 2,24\% e 2,10\%, e a produção volumétrica em 2,92\%, 1,79\% e $1,73 \%$, nos cenários 2,3 e 4 respectivamente. Porém, os cenários 2,3 e 4 obtiveram sucesso no controle de corte de talhões adjacentes, segundo suas restrições, e impedindo a formação de extensas áreas contiguas como encontrado no cenário 1 ( 153,25 ha).

Palavras-chave: Programação linear inteira; restrição URM; restrição ARM.
\end{abstract}

\begin{abstract}
Spatial adjacency constraints effect in optimized forest planning. The objective of the study was to apply the spatial adjacency constraints in the forest harvest scheduling when using the traditional forest planning model. It was analyzed and compared the impact on NPV (Net Present Value) and the volumetric production of wood. The area of study was formed by 52 stands of eucalyptus. A total of 254 forest management alternatives were simulated, where 4 forest harvest scheduling scenarios contained the integer linear programming were created, following the model type I and the maximization of the NPV for 7 years of the horizon planning. Spatial adjacency constraints were applied to scenarios 2 (URM), 3 (ARM50) and 4 (ARM70) but not to scenario 1. The results showed that the spatial adjacency constraints reduced NPV in 3,74\%,2,24\% and 2,10\%, and the volumetric production in $2,92 \%, 1,79 \%$ e $1,73 \%$ through the scenarios 2,3 , and 4 , respectively. Therefore, the scenarios 2,3 , and 4 obtained success in controlling the adjacent harvested stands, according to their restrictions, and avoiding the creation of large and continuous areas such as observed in the scenario 1 $(153,25 \mathrm{ha})$

Keywords: Integer linear programming; URM constraint; ARM constraint.
\end{abstract}

\section{INTRODUÇÃO}

A otimização da agenda de corte na colheita florestal contempla uma série de restrições vinculadas à distância de deslocamento de veículos, custos de transporte e metas de produção e de lucro, formando assim um dos pilaras do planejamento de uma empresa florestal. Esse modelo funcional de planejamento, em muitos cenários, desconsidera as relações espaciais entre unidades de manejo, podendo causar impactos negativos ao ambiente. Por outro lado, a introdução das relações espaciais contribui para a construção de áreas mais resistentes a impactos naturais e/ou antrópicos, promovendo uma proteção ao local. Um estudo realizado por Thompson et al. (1998) comprovou que as restrições de adjacência auxiliam na construção de paisagens mais resistentes à propagação do fogo, quando simulados os riscos de desastres por incêndio. 
Assim, uma visão mais integrada da floresta está ganhando atenção ao longo dos anos, principalmente em se tratando das interações entre a vizinhança das unidades de manejo. Um dos tantos exemplos é encontrado pela American Forest and Paper Association, que incentiva processos de sustentabilidade de florestas nos Estados Unidos, ao restringir o corte de áreas contíguas com mais de 120 acres ou 48,55 ha (GOYCOOLEA et al., 2005). Na literatura, ainda é encontrada uma variedade de limites de área, cuja diversidade está atribuída a questões regionais, políticas, a processos de certificação, ou até mesmo a questões operacionais vinculadas ao equipamento utilizado na colheita florestal. Sessions; Bettinger (2001) citam alguns exemplos como áreas máximas de 49 ha (Oregon, EUA), 17 ha (Califórnia, EUA) e 20 ha (Suécia).

Os debates em prol do controle da colheita em extensas áreas de floresta contribuíram para o surgimento do conceito de proteção de hábitats, conhecido como green up. Segundo McNaugthon et al. (2001), o efeito aplicado dessa medida promove um processo de dispersão da colheita florestal na área durante o planejamento da colheita. Essa dispersão é explicada quando um talhão é selecionado para o corte e, pela regra, seus vizinhos ficam impedidos de serem colhidos durante um determinado período de tempo (green up delay). Como exemplo, tem-se a Nova Zelândia, que restringe o corte de talhões vizinhos durante os 5-10 anos subsequentes. Esse tempo de espera é aguardado para permitir que as árvores alcancem uma altura média de 2-4 metros, liberando assim as áreas vizinhas para o corte (NELSON, 2000). McNaugthon et al. (2001) afirmam que o seu surgimento foi basicamente em virtude da necessidade de redução do impacto da erosão do solo após a colheita florestal. Segundo Mcdill; Braze (2000), o pioneiro no desenvolvimento de restrições de adjacência foi Thompson, em 1973, e seu estudo continha propostas com objetivos múltiplos de produção de madeira e proteção à fauna.

Durante o processo de construção dos modelos envolvendo espacialidade, o uso do sistema de informação geográfica é empregado, porém apenas como suporte na criação das restrições, não participando do processo de seleção das áreas a serem colhidas. Dessa forma, surgiu o conceito de planejamento florestal espacial, em que, na visão de Baskent; Keles (2005), o talhão passa a ser observado segundo o seu tamanho, forma e distribuição dentro da floresta, fato que não ocorre no planejamento florestal tradicional. Bettinger; Sessions (2003) acreditam que, brevemente, o planejamento florestal será chamado de planejamento florestal espacial, tendo como foco as atividades do manejo florestal e políticas ambientais.

Öhman (2001) relata a existência de três linhas distintas de restrições espaciais: a primeira delas considera a conectividade entre talhões/áreas; a segunda executa um processo contrário, o de dispersão da colheita ao longo da floresta; a terceira é uma mistura de características da primeira com a segunda. A restrição de adjacência se enquadra na segunda linha, e ainda pode ser dividida em duas classes distintas: restrições por unidade e por área máxima adjacente de corte (MURRAY, 1999). O autor propõe, de forma bem objetiva, uma classificação de uso dessas terminologias, sendo as restrições do modelo: URM (unit restriction model) e ARM (area restriction model).

Os modelos que apresentam restrições por unidade (URM) desenvolvem uma incompatibilidade de colheita florestal de dois ou mais talhões vizinhos (STEIGUER et al., 2003). A outra opção de restrição (ARM) permite a colheita de talhões vizinhos, desde que a soma das áreas deles não ultrapasse um limite máximo de área preestabelecido (STEIGUER et al., 2003). A diferença entre os modelos contendo ARM e URM é o fato de haver a possibilidade de colher talhões vizinhos (respeitando um limite máximo de área) no primeiro caso, e no segundo caso, não. Além disso, o grau de dificuldade de se formular um modelo ARM é muito maior que no modelo URM (ZHU; BETTINGER, 2007).

A resolução de problemas florestais envolvendo as restrições ARM pelo método exato só surgiu a partir do trabalho de McDill et al. (2002), estimulando assim a abertura para novas descobertas e desenvolvimento de mecanismos de resolução. Contudo, o surgimento e resolução de modelos relacionados às restrições do tipo URM são mais antigos, sendo originários na década de 70. A diferença temporal existente entre o pleno conhecimento dessas restrições demonstra o grau de complexidade e evolução dos problemas florestais. Além das restrições de adjacência, podem-se encontrar ainda restrições designadas para a manutenção de corredores ecológicos (FISCHER; CHURCH, 2003), algumas ligadas a índices de paisagem (HEINONEN et al., 2007), restrições de blocos para facilitar a colheita (NELSON, 2001) e restrições para problemas de green-up (BOSTON; BETTINGER, 2001), dentre outras.

Nelson (2000) considera que o trabalho do planejador florestal é conhecer o impacto econômico dessas restrições na atividade florestal, sendo uma proposta desafiadora e importante. Assim, surgem 
questionamentos: Qual o custo desse tipo de restrição espacial para a indústria de base florestal? Existem alternativas mais vantajosas economicamente e que se enquadram na linha ambiental, como o retalhonamento? Qual a redução volumétrica em projetos dessa natureza? Qual o limite de tolerância aceitável de adjacência no manejo de extensas florestas e em quais anos aplicar? Que tipo de restrição espacial empregar no planejamento florestal? Existem ferramentas para modelar esses problemas? Quais os métodos de resolução? Há ainda uma série de outras questões ligadas diretamente à parte operacional e estratégica do empreendimento florestal.

Dessa maneira, os objetivos deste estudo foram: introduzir restrições espaciais de adjacência no agendamento da colheita florestal, comparando os resultados com a versão tradicional de planejamento florestal por meio do VPL (valor presente líquido) e da produção volumétrica de madeira; verificar as diferenças entre as restrições de adjacência do tipo ARM (area restriction model) e URM (unit restriction model) no problema aplicado; e comparar os efeitos de dois limites de área (50 ha e 70 ha) na restrição do tipo ARM ao serem introduzidas no modelo matemático.

\section{MATERIAL E MÉTODOS}

\section{Área de estudo}

$\mathrm{O}$ planejamento da colheita florestal foi direcionado a 5 fazendas pertencentes na época à empresa VCP - Votorantim Celulose e Papel, atualmente Fibria, sendo todas localizadas no município de Caçapava, SP. A área total destinada ao estudo compreende 1.490,7 ha e contém reflorestamentos com espécies e clones de eucalipto da empresa. A floresta é dividida em 52 talhões ou unidades de manejo, apresentando uma heterogeneidade de idades e áreas. A tabela 1 apresenta a distribuição da floresta por idade, considerando a área e a frequência de talhões.

Tabela 1. Estrutura inicial da floresta destinada ao planejamento da colheita.

Table 1. Initial structure for planning harvesting.

\begin{tabular}{lcccc}
\hline \multirow{2}{*}{ Classe de idade (anos) } & \multicolumn{2}{c}{ Unidade de manejo florestal } & \multicolumn{2}{c}{ Área } \\
\cline { 2 - 5 } & Frequência & $(\boldsymbol{\%})$ & $(\mathbf{h a})$ & $\mathbf{( \% )}$ \\
\hline 1 & 9 & 17,30 & 233,65 & 15,68 \\
2 & 0 & 0,00 & 0,00 & 0,00 \\
3 & 6 & 11,54 & 242,58 & 16,27 \\
4 & 1 & 1,92 & 40,78 & 2,73 \\
5 & 25 & 48,08 & 629,40 & 42,22 \\
6 & 11 & 21,16 & 344,29 & 23,10 \\
\hline Total & 52 & 100,00 & 1490,70 & 100,00 \\
\hline
\end{tabular}

\section{Planejamento tático da colheita florestal}

Agendamento da colheita florestal

$\mathrm{O}$ agendamento da colheita florestal é normalmente parte integrante do manejo florestal e apresenta como objetivos a definição da agenda de corte das unidades, auxiliando na regulação da floresta (HOTVEDT, 1983). Assim, ele pode ser enquadrado dentro do planejamento operacional ou tático da colheita florestal, a depender principalmente do horizonte de trabalho. Geralmente, o planejamento tático apresenta uma escala em torno de 2 a 5 anos do horizonte de planejamento, segundo a espécie manejada, e utiliza alguns resultados do planejamento estratégico (MITCHELL, 2004). Nesse estágio, é definido o tipo de alternativas de manejo adotadas para cada talhão e é realizada uma análise de oferta e demanda do mercado e tipos de produtos desejados pelos consumidores. As metas de produção volumétrica são importantes e devem ser alcançadas nessa fase a partir do agendamento da colheita.

$\mathrm{O}$ agendamento da colheita fornece suporte a uma série de atividades de logística e procedimentos técnicos, em virtude da sequência de unidades de manejo agendadas ao longo do tempo. Assim, o problema florestal teste foi caracterizado com a necessidade de maximizar o valor presente líquido (VPL), seguindo os moldes do modelo tipo I de Johnson; Scheurmann (1977) como referência. Dessa forma, foram introduzidas restrições ligadas à estabilização da produção volumétrica de madeira, o que promove um fornecimento contínuo e constante de madeira dentro de um intervalo predefinido de tempo, regulando a floresta. Além disso, foram empregados dois tipos de restrições espaciais ligadas à 
adjacência entre unidades de manejo, quais sejam, as restrições ARM (area restriction model) e URM (unit restriction model), aplicadas separadamente.

Assim, o problema foi dividido em 4 tipos de cenário, conforme apresentado na tabela 2. Esses problemas descrevem 4 situações distintas, porém apresentando uma função de maximização semelhante. O cenário 1 considera apenas o uso de restrições de controle volumétrico, sendo uma situação em que os cuidados ambientais e a visão de interação entre unidades de manejo não foram explicitamente contemplados pelo modelo. Os cenários 2, 3 e 4 empregaram o uso de restrições de adjacência além da restrição de controle volumétrico. As restrições de integralidade também foram usadas para garantir a seleção de apenas uma única opção de manejo por talhão, estando presente em todos os cenários. Nos cenários 3 e 4, a restrição ARM foi configurada considerando duas opções. A primeira opção estabeleceu um limite máximo de 50 ha (ARM50), ou seja, um grupo de talhões adjacentes apresentando uma área total conjunta limitada a 50 ha. Essa opção adotou um limite próximo ao definido pela American Forest and Paper Association. A segunda opção utilizou uma área máxima de 70 ha (ARM70).

Tabela 2. Cenários estudados com restrições espaciais de adjacência no agendamento da colheita florestal.

Table 2. Studied scenarios with spatial adjacency constraints in forest harvest scheduling.

\begin{tabular}{lcccccc}
\hline Cenários & Planejamento florestal & Volume & Integridade & URM & ARM50 & ARM70 \\
\hline 1 & Tradicional & $\mathrm{x}$ & $\mathrm{x}$ & - & - & - \\
2 & Espacial & $\mathrm{x}$ & $\mathrm{x}$ & $\mathrm{x}$ & - & - \\
3 & Espacial & $\mathrm{x}$ & $\mathrm{x}$ & - & $\mathrm{x}$ & - \\
4 & Espacial & $\mathrm{x}$ & $\mathrm{x}$ & - & - & $\mathrm{x}$ \\
\hline
\end{tabular}

Os quatro cenários tiveram uma demanda de madeira anual e constante de $69.000 \mathrm{~m}^{3}$, com variação de $\pm 42 \%$ de sua meta. A definição dos limites de produção volumétrica foi proposta em virtude de testes preliminares, nos quais uma porcentagem mais conservadora (menor) de desvio prejudicou a comparação dos cenários. O horizonte de planejamento (HP) estudado foi de 7 anos, e a taxa de juros foi fixada em $10 \%$ ao ano em todos os cenários simulados. As projeções de crescimento volumétrico da floresta seguiram dados da própria empresa, assim como os custos de manutenção da floresta, de colheita e transporte. O preço de venda da madeira utilizado foi de $\mathrm{R} \$ 76,00 \mathrm{~m}^{-3}$. O regime de manejo adotado foi o pulp wood, sem considerar reformas no plantio, apenas o corte e replantio sucessivo. Dessa forma, a idade máxima permitida de corte foi estabelecida em 7 anos, e a mínima em 5 anos, para a construção das 254 alternativas de manejo. Ainda foi permitido deixar o talhão ocioso, ou seja, sem definir nenhuma opção de manejo para ele, já que os cenários contendo adjacência foram sensíveis à falta dessa opção, em virtude das características da floresta e de seu estado desregulado.

\section{A vizinhança}

A definição da adjacência entre talhões vizinhos seguiu um processo criterioso, pois a área de estudo é composta por estradas, rodovias, linhas de transmissão de energia elétrica e até um gasoduto. Dessa forma, foram definidos os seguintes critérios de adjacência entre talhões: a) adjacência tradicional: talhões que se tocam lateralmente e dividem uma linha em comum, exceto os adjacentes somente em encruzilhadas de estradas, ou que têm apenas um ponto de contato; b) presença de gasoduto, estradas e de redes de transmissão de energia elétrica: não considerado como uma barreira à adjacência, seguindo o critério anterior; c) presença de rodovias: não foi considerada uma barreira, exatamente para reduzir o impacto visual da colheita na paisagem, devido ao tráfego de veículos e pessoas; d) não adjacentes: todas as unidades que não se encaixaram nos critérios $a, b$ e $c$.

\section{Formulação matemática dos cenários}

O problema de agendamento da colheita florestal testado requer o uso de variáveis binárias $\{0,1\}$, devido à necessidade de colher totalmente uma unidade de manejo, sendo uma exigência do regime de manejo adotado e das restrições de adjacência. Assim, os cenários foram modelados seguindo a estrutura da programação linear inteira para a obtenção das soluções. Dessa maneira, a formulação matemática seguiu a seguinte estrutura: (1) função objetivo dimensionada para maximizar o valor presente líquido (VPL) da floresta; (2) restrição de integridade das unidades de manejo; (3) e (4) 
restrições de controle do fluxo de produção volumétrico de madeira; e (7) restrições binárias das variáveis de decisão do modelo. No cenário 2 foi introduzida a restrição (5), e nos cenários 3 e 4 foi acrescida a restrição (6) e excluída a restrição (5).

Função objetivo: maximizar

$$
\mathrm{VPL}=\sum_{\mathrm{i}=1}^{\mathrm{M}} \sum_{\mathrm{j}=1}^{\mathrm{P}} \mathrm{c}_{\mathrm{ij}} \mathrm{x}_{\mathrm{ij}}
$$

Sujeito a:

$$
\begin{aligned}
& \sum_{j=1}^{P} x_{i j} \leq 1 \\
& \forall_{\mathrm{j}}, \forall_{\mathrm{i}=1,2 \ldots \mathrm{M}} \\
& \sum_{\mathrm{i}=1}^{\mathrm{M}} \sum_{\mathrm{j}=1}^{\mathrm{P}} \mathrm{v}_{\mathrm{ij}} \mathrm{x}_{\mathrm{ijT}} \geq \mathrm{L}_{\text {inferior }} \\
& \sum_{\mathrm{i}=1}^{\mathrm{M}} \sum_{\mathrm{j}=1}^{\mathrm{P}} \mathrm{v}_{\mathrm{ij}} \mathrm{x}_{\mathrm{ijT}} \leq \mathrm{L}_{\text {superior }} \\
& \sum_{\substack{\mathrm{i}=1 \\
\mathrm{i} \neq \mathrm{k}}}^{\mathrm{M}} \mathrm{a}_{\mathrm{ik}} \mathrm{x}_{\mathrm{ijT}}+\left[\sum_{\mathrm{i}=1}^{\mathrm{M}} \mathrm{a}_{\mathrm{ik}}\right] \cdot \mathrm{x}_{\mathrm{ijT}} \leq \sum_{\mathrm{i}=1}^{\mathrm{M}} \mathrm{a}_{\mathrm{ik}} \\
& \sum_{\mathrm{U} \in \mathrm{P}_{\mathrm{I}}} \mathrm{x}_{\mathrm{Ut}} \leq \mathrm{n}_{\mathrm{P}_{1}}-1 \\
& \forall_{\mathrm{i}}, \forall_{\mathrm{j}}, \forall_{\mathrm{T}=1,2, \ldots \mathrm{HP}}, \forall_{\mathrm{i}}, \forall_{\mathrm{j}}, \forall_{\mathrm{T}=1,2, \ldots \mathrm{HP}}(3 \\
& \forall_{\mathrm{i}}, \forall_{\mathrm{j}}, \forall_{\mathrm{T}=1,2, \ldots \mathrm{HP}}, \forall_{\mathrm{i}}, \forall_{\mathrm{j}}, \forall_{\mathrm{T}=1,2, \ldots \mathrm{HP}}(4) \\
& \mathrm{x}_{\mathrm{ij}} \in\{0,1\} \\
& \forall_{\mathrm{i}}, \forall_{k}, \forall_{\mathrm{j}}, \forall_{\mathrm{T}=1,2, \ldots \mathrm{HP}}(5) \\
& \forall_{P_{i}} \text { e } \mathrm{t}=1,2,3, \ldots, \mathrm{T}(6)
\end{aligned}
$$

Em que: $\mathrm{M}=$ número total de unidades de manejo;

$\mathrm{P}=$ número total de alternativas de manejo;

$\mathrm{x}_{\mathrm{ij}}=$ variável binária (0/1) de decisão do modelo, referente ao talhão $i$ e alternativa de manejo $j$;

$\mathrm{c}_{\mathrm{ij}}=$ valor presente líquido pertencente ao talhão $i$ segundo a alternativa de manejo $j$;

$\mathrm{T}=$ período total de tempo de planejamento, em anos;

$\mathrm{v}_{\mathrm{ij}}=$ volume total produzido no talhão $i$ pela alternativa de manejo $j$;

$\mathrm{U}=$ unidade de manejo ou talhão;

$\mathrm{k}=$ talhão número;

$\mathrm{a}_{\mathrm{ik}}=$ matriz binária $\{0,1\}$ de adjacência entre talhões, onde $i=k$;

$\mathrm{P}_{\mathrm{i}}=$ conjunto de talhões adjacentes, ou grupo;

$\mathrm{n}_{\mathrm{Pi}}=$ número de talhões adjacentes presentes no conjunto, ou grupo.

A restrição de adjacência do tipo URM foi formulada seguindo a mesma lógica encontrada nos trabalhos de Alonso (2003) e Castro (2007). Já a construção das restrições de adjacência do tipo ARM seguiu a estrutura apresentada por McDill et al. (2002), utilizando-se ainda a implementação do path algorithm desenvolvido pelo mesmo autor para a identificação dos grupos de unidades de manejo adjacentes. A sequência a seguir descreve esse algoritmo:

- Inicie com um par de talhões adjacentes qualquer. Se o somatório em área exceder o máximo permitido, então escreva a restrição para esse conjunto e selecione outro par qualquer. Caso contrário, continue os passos;

- Selecione qualquer talhão adjacente ao grupo formado anteriormente, adicionando-o. Então, calcule a área acumulada;

- Defina uma rede, baseada nesse grupo, na qual um nó corresponde a cada talhão e um arco é gerado para conectá-los. Identifique cada possibilidade de gerar uma nova ligação ao grupo já formado. A cada excedente de área acumulada formada, uma nova restrição é gerada, verificando-se sempre o não fechamento do ciclo, ou seja, se um novo talhão adicionado não se encontra presente no grupo formado. A presença de múltiplas ramificações é comum, e deve ser seguida. Além disso, devem-se eliminar as restrições repetidas;

- Se todos os grupos forem identificados e convertidos em restrições ARM, pare. Caso contrário, volte ao passo 2 .

\section{Resolução e processamento}

A construção dos modelos envolvendo os quatro cenários foi gerada utilizando-se a linguagem de programação Visual Basic ${ }^{\circledR}$ versão 6.0 Enterprise, da Microsoft ${ }^{\circledR}$, licenciada para o PPGEF (Programa 
de Pós-Graduação em Engenharia Florestal) da UFPR. Os modelos matemáticos de PLI (programação linear inteira) foram escritos por essa ferramenta, porém o seu processamento foi realizado através do software LINGO 9.0 (Language for Interactive General Optimizer). O processamento foi executado em um computador com processador Celeron ${ }^{\circledR}$ core 2 duo $2100 \mathrm{MHz}$ e 2 Gb de RAM.

\section{RESULTADOS E DISCUSSÃO}

A formulação dos modelos contendo restrições de adjacência foi mais complexa de implementar, quando comparada com o processo tradicional de planejamento. Essa dificuldade é observada no número de restrições presentes em cada cenário, tendo em vista a necessidade de identificar todas as relações espaciais entre as unidades de manejo. A diferença no número de restrições entre esses dois tipos de abordagem de planejamento foi considerada alta. No cenário 1, onde foi aplicado o planejamento florestal tradicional, foram utilizadas apenas 66 restrições; já nos cenário 2, 3 e 4 foram geradas, respectivamente, 250, 422 e 580 restrições, o que representa aumentos de 379\%, 639\%, e 879\%. Os cenários contendo as restrições ARM (cenários 3 e 4) apresentaram um número maior de restrições que o modelo envolvendo o uso de restrições do tipo URM (cenário 2). A razão dessa diferença foi o maior número de combinações existentes entre unidades de manejo, as quais poderiam ser selecionadas para a colheita sem desrespeitar o limite máximo imposto pela restrição ARM. A mesma lógica foi observada ao se aumentar o limite de 50 ha para 70 ha na restrição ARM.

No estudo desenvolvido por McDill et al. (2002), as restrição de adjacência do tipo ARM também apresentaram um maior número frente às restrições do tipo URM. Além disso, os autores comentam que o tamanho e a complexidade dos modelos envolvendo o uso de restrições de adjacência ARM aumentam na medida em que são disponibilizados novos talhões para a colheita, no mesmo período de tempo.

Analisando o tempo de processamento dos 4 cenários, que foi de $6.898 \mathrm{~s}$ no cenário $1,32 \mathrm{~s}$ no cenário 2, 44 s no cenário 3 e 376 s no cenário 4, pode-se observar que o número elevado de restrições de adjacência não afetou o tempo de processamento via PLI, pelo contrário, permitiu que o algoritmo branch-and-bound encontrasse solução ótima mais rapidamente. O cenário 1, considerado o mais simples, foi o mais exigente em tempo de processamento, e uma possível resposta para o fato está no maior número de soluções inteiras factíveis e próximas encontradas, uma vez que reduzidas melhoras proporcionam um aumento na busca do algoritmo branch-and-bound. Entretanto, segundo McDill et al. (2002), o tamanho dos modelos geralmente influencia esse aspecto, sendo que os modelos envolvendo as restrições do tipo ARM são mais dispendiosos de tempo que os do tipo URM. A mesma tendência foi observada no presente estudo.

Bettinger et al. (1999) formularam um modelo composto por restrições de adjacência URM e de controle do fluxo de produção de madeira/período, com o propósito de maximizar a produção volumétrica de madeira, a partir da programação linear (PL) relaxada, PLI e busca tabu. A área de estudo dos autores abrangia duas florestas de Douglas-fir (Pseudotsuga menziesii), com 40 e 700 talhões, nos EUA. No estudo dos autores, a floresta composta por 40 talhões obteve um ótimo global via PL relaxada em 9 segundos e uma média de 6,5 minutos para a meta-heurística. Entretanto, utilizando a PLI não foi possível obter o ótimo global até o limite de 5 horas, e sim uma solução factível. No caso da floresta com 700 talhões, outro cenário desenvolvido pelos autores, não foi possível encontrar uma solução factível via PLI, porém, pela meta-heurística, essa operação obteve sucesso em 215 minutos, indicando uma solução factível.

O cenário 4 foi o mais dispendioso de tempo de processamento, quando comparado com os cenários 2 e 3 envolvendo adjacência, porém apresentando um número maior de restrições. A explicação para o fato é exatamente por ser mais flexível na questão da adjacência e por isso apresentar um maior número de combinações factíveis de solução. Os casos 2 e 3 , por outro lado, tiveram respostas de tempo semelhantes entre si, devido à maior rigidez de suas restrições espaciais de adjacência. Alonso (2003) aplicou as restrições URM em 5 cenários florestais simulados de Pinus, com o propósito de maximizar a receita e suprir a demanda dos produtos gerados. Com isso, os tempos de processamento via PLI foram de 23 segundos (15 talhões e HP de 10 anos), 7 segundos (25 talhões e HP de 15 anos), 4 segundos (32 talhões e HP de 15 anos) e 6 segundos (50 talhões e HP de 30 anos). A velocidade na resolução deve-se à simplicidade dos problemas formulados. 
O valor do VPL foi influenciado pelo tipo de abordagem de planejamento executado, no qual reduções de seus valores podem ser percebidas à medida que o modelo se torna mais restritivo à questão da adjacência. Assim, o desvio percentual de perda do VPL ao utilizar o modelo de planejamento florestal espacial (cenários 2, 3 e 4) ficou abaixo de 4\%, ou, de forma mais detalhada: 3,74\% (cenário 2), 2,24\% (cenário 3) e 2,10\% (cenário 4). Entretanto, vale lembrar que essa tendência encontrada não é uma regra geral, pois seus resultados são dependentes do tipo de relações espaciais de adjacência existente em cada área.

Hoganson; Borges (1999) constataram uma redução na produção volumétrica e no VPL contendo as restrições de adjacência (URM). Nos cenários estudados pelos autores, a perda final no VPL chegou a $1,3 \%, 1,4 \%, 1,5 \%$ e $1,7 \%$, referente à introdução dessas restrições em cada década do horizonte de planejamento. Os menores valores de perda encontrados estão vinculados à presença de períodos dentro do HP, nos quais foi permitida a colheita de talhões adjacentes, fato não ocorrido no presente estudo. McDill et al. (2002) comentam que os modelos formulados com restrições do tipo ARM geram os maiores valores nas funções de lucro, quando comparados com o modelo utilizando restrições do tipo URM. O mesmo resultado foi encontrado no presente estudo.

Carter et al. (1997), estudando os efeitos das restrições de adjacência (URM) nos modelos de planejamento florestal, constataram reduções de seus valores de VPL quando aplicados em uma área natural de Pinus, localizada na Flórida (EUA), composta por 937 talhões. As reduções apresentadas foram na ordem de $18,1 \%$ a $36,6 \%$, a depender do cenário estabelecido, afetando também a produção volumétrica de madeira. Segundo os autores, a explicação para uma redução dessa magnitude foi relacionada à maior conexão entre talhões na vizinhança, ou seja, uma elevada interação espacial entre os talhões adjacentes. A confirmação dessa afirmativa está no número de violações da adjacência (723 ocorrências) pelo modelo sem o uso dessa restrição.

Alonso (2003) encontrou o custo de aplicar a restrição URM. Esse valor ficou na casa dos $2 \%$ em uma floresta hipotética de Pinus composta de 15 talhões. Já em uma floresta de eucalipto dividida em 105 talhões o desvio foi de 6,6\%, no trabalho de Castro (2006).

A redução dos impactos ocasionados pelas restrições de adjacência (URM) pode ser mitigada da seguinte maneira: estipular uma regra na formulação dos modelos de planejamento, no qual os talhões com maior área tenham preferência na seleção; permitir um limite mínimo de violação dessa restrição; e alterar a estrutura de vizinhança entre os talhões (NELSON, 2000). Entretanto uma medida possível de se aplicar é por meio de formulações matemáticas similares às dos cenários 3 e 4, testadas no presente estudo.

As perdas volumétricas totais observadas nos cenários com restrições espaciais ficaram abaixo de 3\%, quando comparadas ao planejamento florestal tradicional. Os valores absolutos da produção volumétrica foram de 614.919,46 $\mathrm{m}^{3}$ (cenário 1), 596.964,4 $\mathrm{m}^{3}$ (cenário 2), 603.937,3 $\mathrm{m}^{3}$ (cenário 3) e $604.279,2 \mathrm{~m}^{3}$ (cenário 4). Já em termos percentuais essas perdas frente ao cenário 1 foram de 2,92\% (cenário 2), 1,79\% (cenário 3) e 1,73\% (cenário 4). Marianov et al. (2004) estudaram uma floresta hipotética composta por 144 grids, adotando a programação multiobjetivo com o propósito de maximizar a produção volumétrica e a preservação de espécies. Nesse estudo, a redução volumétrica de madeira chegou a 38\% quando introduzidas restrições de adjacência, em virtude da forte interação entre vizinhanças.

Analisando o número de grupos formados por unidades de manejo, após o agendamento da colheita, percebeu-se a formação de áreas contíguas nos cenários 1,3 e 4, conforme mostra a tabela 3 . $\mathrm{O}$ tamanho das áreas contíguas colhidas esboça o sucesso no controle espacial, pelos cenários destinados a essa causa, já que os limites de 50 ha (ARM50) e 70 ha (ARM70) foram devidamente respeitados, bem como o uso das restrições do tipo URM. Assim, o não uso dessas restrições promoveu o surgimento de áreas contíguas máximas iguais a 153,95 ha no segundo e sétimo ano de agendamento, apresentando ainda a formação de 5 grupos com área contígua superior a 90 ha, ao longo do período de planejamento.

Nelson (2000) afirma que o uso de restrições de adjacência promove uma maior dispersão da atividade de colheita na área, resultando no aumento dos gastos na construção de estradas ao longo do horizonte de planejamento. Essa mesma dispersão foi observada, porém infelizmente não foram analisados os custos ligados a deslocamentos na colheita para os cenários formulados.

No trabalho de Boston; Bettinger (2001), encontram-se referências sobre a decisão de considerar o uso das restrições de adjacência no agendamento da colheita florestal. Os autores concluíram que uma 
produção volumétrica de madeira elevada e/ou preços dos produtos florestais atraentes fazem com que o uso de restrições de adjacência não seja adotado. Porém quando estes efeitos ocorrem em direções opostas, os incentivos econômicos para a adoção das restrições de adjacência tornam-se recomendados.

Tabela 3. Áreas e grupos adjacentes formados após o agendamento da colheita florestal, considerando os 4 cenários propostos.

Table 3. Areas and adjacent groups formed after the forest harvest scheduling considering the 4 scenarios proposed.

\begin{tabular}{|c|c|c|c|c|c|c|c|c|}
\hline \multirow{2}{*}{ Cenários } & \multirow{2}{*}{ Adjacência } & \multicolumn{7}{|c|}{ Horizonte de planejamento } \\
\hline & & 1 & 2 & 3 & 4 & 5 & 6 & 7 \\
\hline \multirow[t]{2}{*}{1} & Áreas (ha) & 95,22 & $\begin{array}{c}153,25 \\
70,05\end{array}$ & 137,02 & 67,78 & 60,93 & $\begin{array}{c}52,59 ; 86,76 \\
33,83\end{array}$ & $\begin{array}{c}153,95 ; 30,95 ; \\
116,46\end{array}$ \\
\hline & Grupos & 1 & 2 & 1 & 1 & 1 & 3 & 3 \\
\hline \multirow{2}{*}{2} & Áreas & 0 & 0 & 0 & 0 & 0 & 0 & 0 \\
\hline & Grupos & 0 & 0 & 0 & 0 & 0 & 0 & 0 \\
\hline \multirow{2}{*}{3} & Áreas & $\begin{array}{l}47,04 \\
43,86\end{array}$ & $\begin{array}{l}41,43 \\
47,43\end{array}$ & $\begin{array}{l}20,21 \\
25,26\end{array}$ & 0 & 46,39 & 47,$04 ; 43,86$ & 47,83 \\
\hline & Grupos & 2 & 2 & 2 & 0 & 1 & 2 & 1 \\
\hline \multirow[t]{2}{*}{4} & Áreas & $\begin{array}{l}52,59 \\
64,27\end{array}$ & $\begin{array}{l}56,08 \\
37,93\end{array}$ & $\begin{array}{l}58,7 \\
53,92\end{array}$ & 67,79 & 60,93 & 61,78 & $\begin{array}{c}56,08 ; 41,43 \\
47,83 ; 37,93 \\
30,95\end{array}$ \\
\hline & Grupos & 2 & 2 & 2 & 1 & 1 & 1 & 5 \\
\hline
\end{tabular}

Fixando o agendamento da colheita no segundo ano de planejamento, percebeu-se que as produções volumétricas de madeira apresentaram valores muito próximos (cenário $1-97.961,91 \mathrm{~m}^{3}$; cenário 2 - 97.809,37 $\mathrm{m}^{3}$; cenário $3-97.970,01 \mathrm{~m}^{3}$; cenário 4 - 97.685,59 $\mathrm{m}^{3}$ ), bem como a área total designada à colheita florestal (cenário 1 - 325,81 ha; cenário 2 - 323,98 ha; cenário 3 - 324,38 há; cenário 4 - 328,32 ha). O número de talhões selecionados para o corte foi praticamente o mesmo, exceto para o cenário 3, diferenciando-se no conjunto de unidades de manejo selecionados para o agendamento, conforme tabela 4. As áreas marcadas na tabela 4 indicam a igualdade de talhões selecionados nos cenários 2,3 e 4 frente ao cenário 1 .

Tabela 4. Lista de talhões destinados à colheita florestal envolvendo os 4 cenários propostos e o segundo ano do horizonte de planejamento.

Table 4. List of the stands selected to the forest harvest considering the 4 scenarios and the second year of the planning horizon.

\begin{tabular}{lllllllllllll}
\hline Cenários & \multicolumn{10}{c}{ Talhões selecionados para a colheita florestal no segundo ano } \\
\hline 1 & 2 & 4 & 5 & 6 & 7 & 9 & 18 & 21 & 23 & 29 & 38 & 39 \\
2 & 3 & 7 & 9 & 12 & 13 & 17 & 19 & 22 & 25 & 28 & 30 & 39 \\
3 & 2 & 6 & 8 & 9 & 15 & 17 & 18 & 22 & 28 & 30 & 39 & - \\
4 & 2 & 3 & 6 & 9 & 18 & 20 & 21 & 22 & 26 & 27 & 36 & 39 \\
\hline
\end{tabular}

Assim, do ponto de vista técnico-operacional, esses resultados mostram uma aproximação entre os cenários, para a atividade da colheita florestal. Contudo, ao executar a colheita desses talhões para o ano analisado e nos diferentes cenários abordados, notou-se que seus efeitos foram diferentes na matriz paisagística, conforme apresentado na figura 1. O planejamento florestal tradicional (cenário 1) definiu uma sequência de colheita que incluiu talhões adjacentes, proporcionando a formação de 2 grupos de talhões adjacentes com áreas de 153,95 ha e 70,05 ha. Por sua vez, os casos contemplando o planejamento florestal espacial (cenários 2, 3 e 4) não apresentaram grupos de talhões adjacentes superiores ao mínimo estabelecido, não chegando a constituir a formação desses grupos no cenário 2, conforme esperado, por se tratar de um modelo tipo URM. Nos cenários 3 e 4, a presença de grupos de talhões adjacentes ocorreu, porém devidamente controlada pelo limite de área permitido. Sendo assim, o cenário 3 permitiu a formação de 2 grupos de talhões adjacentes, com áreas de 47,83 ha e 41,43 ha, enquanto que o cenário 4, analogamente, gerou o mesmo número de grupos, porém com áreas de 37,93 ha e 56,08 ha. 


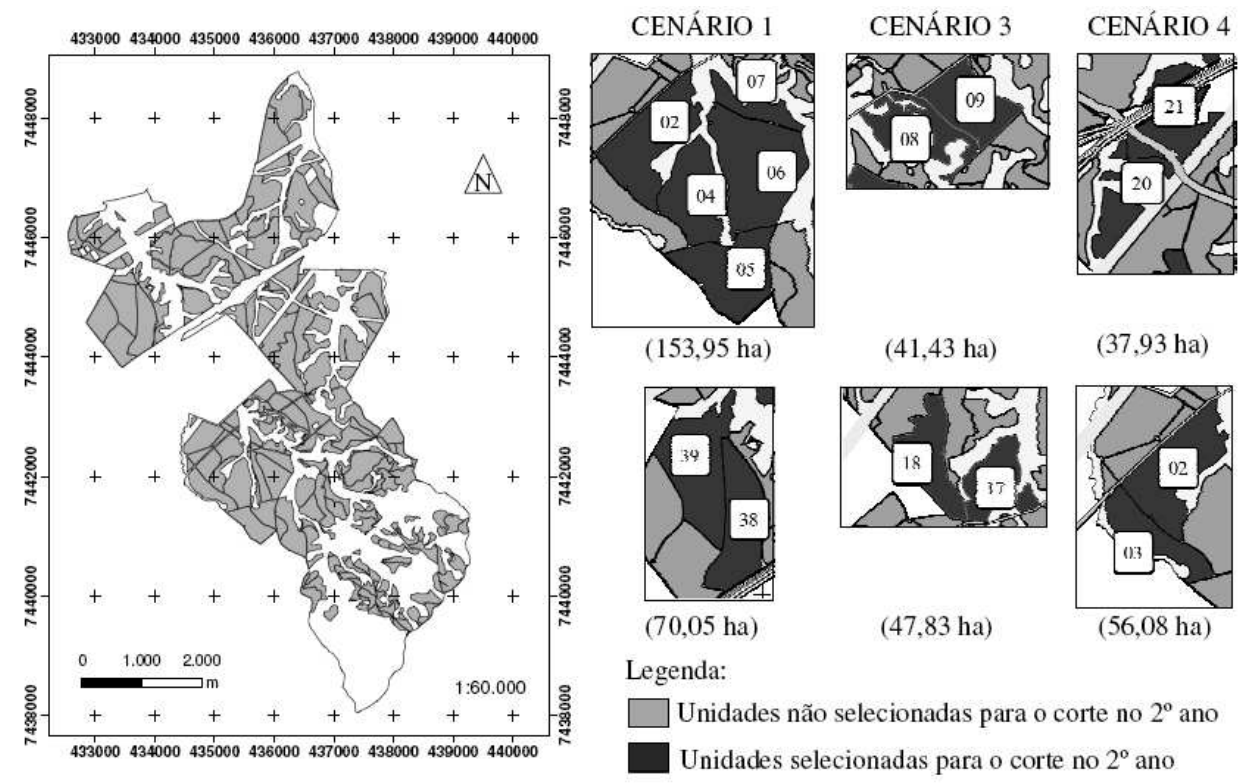

Figura 1. Estrutura espacial formada no segundo ano do agendamento da colheita florestal, considerando apenas a identificação de grupos de talhões adjacentes e suas áreas nos cenários 1, 3 e 4 .

Figure 1. Spatial structure designed in the second year of the harvest scheduling problem considering only the identification of the adjacent stands groups and their areas in scenarios 1, $3 \mathrm{e} 4$.

O uso das restrições de adjacência acabou por deixar alguns talhões ociosos ao final do período de planejamento estabelecido. Dessa maneira, quando esse tipo de evento ocorre em florestas nativas, devido ao uso de restrições de adjacência, muitas vezes os talhões são abandonados para virar reserva (MARIANOV et al., 2004). Entretanto, devido ao grande investimento realizado em florestas plantadas e às expectativas de retorno do capital, o abandono de talhões torna-se um assunto delicado. Além disso, de modo geral no Brasil, as florestas de eucalipto possuem altos incrementos volumétricos, e o abandono de um talhão significa a não-intervenção na competição entre árvores, resultando no processo de morte natural e redução do crescimento no povoamento. Por outro lado, a não-introdução dessas restrições no cenário 1 proporcionou a seleção de uma alternativa de manejo para cada unidade de manejo, promovendo uma intervenção em toda a floresta ao longo dos 7 anos.

Os talhões considerados problemáticos na questão adjacência foram os identificados como $15 \mathrm{e}$ 38 no cenário 2, e 38 nos cenários 3 e 4 . O maior número de unidades problemáticas no cenário 2 deveuse ao uso das restrições de adjacência do tipo URM, que são mais rígidas nessa questão. Uma medida para evitar a formação desses talhões é o processo de retalhonamento, o que proporciona a formação de novas configurações espaciais na floresta. Assim, os maiores talhões são subdivididos em unidades menores, para formar talhões novos. A outra opção é aumentar o limite de área na restrição ARM, mas essa medida não faz sentido, pois, com esse aumento, o agendamento da colheita passa a incluir mais talhões adjacentes, a ponto da restrição ARM ser inoperante no modelo.

\section{CONCLUSÕES}

- O modelo de planejamento florestal espacial é apto e viável ao uso, e as restrições de adjacência URM, ARM50 e ARM70, formuladas e implementadas na presente pesquisa, contribuem para o controle das relações espaciais entre talhões adjacentes.

- O uso de restrições de adjacência reduz o valor do VPL, de acordo com cada tipo de restrição de adjacência, sendo 3,74\% URM (cenário 2), 2,24\% ARM50 (cenário 3) e 2,20\% ARM70 (cenário 4), o que pode ser observado com a produção volumétrica. 
- O aumento no limite de área na restrição de adjacência ARM se traduz em um aumento na produção volumétrica total, bem como melhora no valor do VPL. O uso de restrições URM causa maiores reduções tanto no VPL quanto na produção volumétrica de madeira, quando comparado às restrições ARM50 e ARM70.

- O número de restrições formuladas utilizando as restrições ARM50 é menor que a opção ARM70, porém superior à restrição de adjacência URM.

\section{AGRADECIMENTOS}

Os autores agradecem pelo apoio financeiro e pela disponibilidade dos dados ao pessoal técnico da empresa Votorantim Celulose e Papel S/A, atualmente Fibria: Eng. Davi Rodrigues de Souza, Eng. Honório Kanegae Júnior, Eng. Márcio Barbosa da Conceição e os funcionários José Carlos da Rosa e Reginaldo Soares de Freitas.

\section{REFERÊNCIAS}

ALONSO, L. R. L. O problema da consideraçião de restrições de adjacência em um planejamento florestal. 114 f. Dissertação (Mestrado em Métodos Numéricos em Engenharia) - Setor de Tecnologia e Ciências Exatas, Universidade Federal do Paraná, Curitiba. 2003.

CARTER, D. R.; VOGIATZIS, M.; MOSS, C. B.; ARVANITIS, L. G. Ecosystem management of infeasible guidelines? Implications of adjacency restrictions for wildlife habitat and timber production. Canadian Journal of Forest Research, Ottawa, n. 27, p. 1302-1310, 1997.

CASTRO, R. R. Regulação de florestas eqüiâneas incluindo restrições de adjacência. 64 f. Dissertação Mestrado) - Universidade Federal de Viçosa, Viçosa, MG, 2007.

BASKENT, E. Z.; KELES, S. Spatial forest planning: A review. Ecological Modelling, Amsterdam, v. 188, p. 29, 2005.

BETTINGER, P.; BOSTON, K.; SESSIONS, J. Intensifying a heuristic forest harvest scheduling search procedure with 2-opt decision choices. Canadian Journal of Forest Research, Ottawa, v. 29, n. 11, p. 1784-1792, 1999.

BETTINGER, P.; SESSIONS, J. Spatial forest planning: to adopt, or not to adopt? Journal of Forestry, Washington, v. 101, n. 2, p. 24-29, 2003.

BOSTON, K.; BETTINGER, P. The economic impact of green-up constraints in the southeastern United States. Forest Ecology and Management, Amsterdam, v. 145, n. 3, p. 191-202, 2001.

FISCHER, D. T.; CHURCH, R. L. Clustering and compactness in reserve site selection: an extension of the biodiversity management area selection model. Forest Science, Bethesda, v. 49, n. 4, 2003.

GOYCOOLEA, M.; MURRAY, A. T.; BARAHONA, F.; EPSTEIN, R.; WEINTRAUB, A. Harvest scheduling subject to maximum area restrictions: exploring the exact approaches. Operational Research, v. 53, n. 3, p. 490-500. 2005.

HEINONEN, T. Developing spatial optimization in forest planning. $48 \mathrm{f}$. Dissertation. University of Joensuu, Joensuu. 2007.

HOGANSON, H. M.; BORGES, J. G. Using dynamic programming and overlapping subproblems to address adjacency in large harvest scheduling problem. Forest Science, Bethesda, v. 44, n. 4, p. 526-538, 1998.

HOTVEDT, J. E. Application of linear goal programming to forest harvest scheduling. Southern Journal of Agricultural Economics, USA, v. 15, p. 103-108, 1983.

JOHNSON, K. N.; SCHEURMANN, H. L. Techniques for prescribing optimal timber harvest and investment under different objectives - discussion and synthesis. Forest Science. Monograph, Washington, v. 18, n. 1, p. 1-31, 1977. 
MARIANOV, V.; SNYDER, S.; REVELLE, C. Trading off species protection and timber production in forests managed for multiple objectives. England. Environment and Planning B, London, v.31, p. 847 - 862. 2004.

MCDILL, M. E.; BRAZE, J. Comparing adjacency constraint formulation for randomly generated forest planning problems with four age-class distributions. Forest Science, Bethesda, v. 46, n. 3, p. 423-436, 2000 .

MCDILL, M. E.; REBAIN, S. A.; BRAZE, J. Harvest scheduling with area-based adjacency constraint. Forest Science, Bethesda, v. 48, n. 4, p. 631-642, 2002.

MCNAUGHTON, A. J.; PAGE, G. D.; RYAN, D. M. 2001. Adjacency Constraints in Forest Harvesting. In: PROCEEDINGS OF THE ORSNZ. Proceedings... New Zealand. 2001, p. 9-15.

MITCHELL, S. A. Operational Forest Harvest Scheduling Optimisation - A mathematical model and solution strategy. 252 f. Thesis. University of Auckland, Auckland. 2004.

MURRAY, A. T. Spatial restriction in harvest scheduling. Forest Science, Bethesda, v. 45, n. 1, p. 45-52, 1999.

NELSON, J. D. The Mechanics of Adjacency. ATLAS/SIMFOR Project Extension Report. p. 8. 2000.

Disponível em: <http://www.forestry.ubc.ca/atlas-simfor/extension/reports.html>. Acesso em: 07/07/2008.

NELSON, J. D. Assessment of forest harvest blocks generated from operational polygons and forestcover polygons in tactical and strategic planning. Canadian Journal of Forest Research, Ottawa, v. 31, p. 682-693, 2001.

ÖHMAN, K. Forest Planning with Consideration to Spatial Relationships. 32 f. Thesis. Swedish University of Agricultural Science, Ultuna. 2001.

SESSIONS, J.; BETTINGER, P. Hierarquical planning: Pathway to the future? In: PROCEEDINGS OF THE FIRST INTERNATIONAL PRECISION FORESTRY COOPERATIVE SYMPOSIUM. Proceedings...Washington. 2001. p. 185-190.

STEIGUER, J. E.; LIBERTI, L.; SCHULER, A.; HANSEN, B. Multicriteria decision models for forestry and natural resources management: an annotated bibliography. 07. Newtown Square, PA: U.S. Department of Agriculture, Forest Service, Northeastern Research Station. 2003. p. 32. (General Technical Report. NE-3).

THOMPSON, W. A.; VERTINSKY, I.; SCHEIER, H.; BLACKWELL, B. A. Using Forest Fire Hazard Modelling in Multiple Use Forest Management Planning. Bulletin of Sustainable Forest Management Network, n.11, p. 1-22, 1998.

ZHU, J.; BETTINGER, P.; LI, R. Additional insight into the performance of a new heuristic for solving spatially constrained forest planning problems. Silva Fennica, Helsinki, v. 41, n. 4, p. 687-698, 2007. 
